\title{
A self-organized resource allocation scheme for heterogeneous macro-femto networks
}

\author{
Mahima Mehta ${ }^{1}$, Nirbhay Rane ${ }^{1}$, Abhay Karandikar ${ }^{1}$, Muhammad Ali Imran $^{2}$ and \\ Barry G. Evans ${ }^{2}$ \\ ${ }^{1}$ Department of Electrical Engineering, Indian Institute of Technology Bombay, Mumbai, India \\ ${ }^{2}$ CCSR, University of Surrey, Guildford, U.K.
}

\begin{abstract}
This paper investigates the radio resource management (RRM) issues in a heterogeneous macro-femto network. The objective of femto deployment is to improve coverage, capacity, and experienced quality of service of indoor users. The location and density of user-deployed femtos is not known a-priori. This makes interference management crucial. In particular, with co-channel allocation (to improve resource utilization efficiency), RRM becomes involved because of both cross-layer and co-layer interference. In this paper, we review the resource allocation strategies available in the literature for heterogeneous macro-femto network. Then, we propose a self-organized resource allocation (SO-RA) scheme for an orthogonal frequency division multiple access based macro-femto network to mitigate co-layer interference in the downlink transmission. We compare its performance with the existing schemes like Reuse-1, adaptive frequency reuse (AFR), and AFR with power control (one of our proposed modification to AFR approach) in terms of 10 percentile user throughput and fairness to femto users. The performance of AFR with power control scheme matches closely with Reuse-1, while the SO-RA scheme achieves improved throughput and fairness performance. SO-RA scheme ensures minimum throughput guarantee to all femto users and exhibits better performance than the existing state-of-the-art resource allocation schemes. Copyright (C) 2014 John Wiley \& Sons, Ltd.
\end{abstract}

KEYWORDS

co-layer interference; femtocell; macrocell; OFDMA; resource allocation

*Correspondence

Mahima Mehta, Department of Electrical Engineering, Indian Institute of Technology Bombay, Mumbai, India.

E-mail: mahima@iitb.ac.in

\section{INTRODUCTION}

With the rapid increase in varied wireless applications and the advent of smart-phones, personal digital assistants, etc., there is a tremendous proliferation in indoor voice and data traffic. It is envisaged that in future, about $50 \%$ of voice traffic and $70 \%$ of data traffic will originate from indoor wireless users [1]. However, there are large penetration losses and attenuation indoors due to which the indoor users often suffer from quality of service (QoS) degradation [1]. To meet the high data rate requirement, efficient mechanisms for resource allocation and interference mitigation indoors are needed. Femto Base Station (BS) deployment is one such mechanism to meet these objectives. It is a short-range, user-deployed, and low-power node operating in the licensed spectrum. It connects mobile devices to a cellular operator's network using residential digital subscriber lines/wired broadband connections [2]. The purpose of femto deployment is to improve capacity (by achieving higher rates due to the proximity to indoor users and increasing reuse of resources) and coverage (by covering the dead zones formed because of insufficient macro signal penetration) in the indoor environment. Because of power-efficient transmission, femtocell network improves battery life and contributes to greener communication. Moreover, femto offloads indoor traffic from the macrocell, which increases capacity of macro BS and reduces capital expenditure (CAPEX) and operational expenditure (OPEX) of network operator. Thus, macro-femto networks are beneficial to both operator and subscribers.

Femtocell network is an overlay deployment (Figure 1) by the indoor users and resource allocation is done independently by macro and femto BSs. This makes radio resource management (RRM) in a macro-femto network challenging. As macrocells and femtocells share the available radio resources, it may cause cross-layer interference (between femtocell and macrocell) and co-layer 


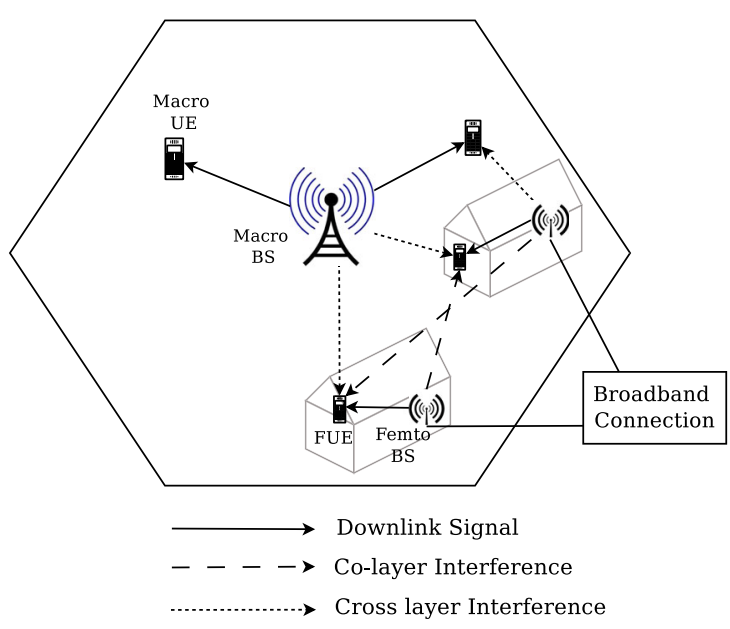

Figure 1. Femtocell overlaid on existing macro-cell network.

interference (between neighboring femtocells) [3]. Ideally, orthogonal resource allocation alleviates cross-layer interference but results in poor resource utilization efficiency. Therefore, co-channel allocation is preferred. Various cross-layer interference reduction schemes are available in the literature [4-6]. However, limited literature is available to address the problem of co-layer interference. In [7] and [8], authors have proposed schemes, which begin with an orthogonal allocation amongst the co-layer femtocells. Then, they apply different variants of adaptive reuse schemes to increase resource utilization efficiency based on either power control or coordination between neighboring femto BSs.

In general, cross-layer interference mitigation has been addressed sufficiently well in the literature. Therefore, we assume in this paper that the cross-layer interference is mitigated by using one of the well illustrated schemes [9] and focus on co-layer interference mitigation only. However, our proposed self-organized resource allocation (SO-RA) scheme reduces cross-layer interference in implicit manner as described in Section 3.3. Reviewing the different schemes available in the literature (discussed in Section 2), it is realized that coordination between neighboring femto $\mathrm{BSs}$ is essential to adapt the resource allocation strategy intelligently (according to the changes in interference levels). With this motivation, we propose a SO-RA scheme for heterogeneous macro-femto network, which mitigates colayer interference. The distinct feature of our scheme is that in addition to reduced co-layer interference, it ensures fairness and improves 10 percentile throughput performance for femto users. It is to be noted that although we illustrate our proposed scheme in the framework of an orthogonal frequency division multiple access (OFDMA)-based Long Term Evolution (LTE) network, it is applicable to any cellular system in general. Also, our proposed scheme provides a generalized framework of self-organized resource allocation, which can be applied to any small-cell network. The only distinction would be the interface used to exchange information between the small cells.
The outline of this paper is as follows: Section 2 discusses few significant approaches available in the literature for interference management in macro-femto networks. Section 3 explains the system model and describes our proposed resource allocation schemes. One is the adaptive frequency reuse (AFR) with power control, which is our proposed modification to AFR approach. Other is the SORA scheme. Section 4 discusses the simulation results and inferences. Finally, we conclude in Section 5.

\section{RELATED WORK}

An overview of interference analysis and resource allocation approaches in a macro-femto network is given in [3]. The fundamental trade-off in achieving interference management in macro-femto networks is to ensure two things: (i) interference due to femto BS does not severely affect macro user equipments (MUEs) and neighboring femto UEs (FUEs) and (ii) the transmit power of femto BS must be sufficiently high to ensure that the rate requirement of FUEs are met.

A centralized approach is one of the ways to achieve this trade-off. In this approach, a centralized controller uses information from femto BSs and FUEs to mitigate cross and co-layer interference. However, because of the random variations in topology and large number of femto BSs, centralized approach may not be scalable. Another approach could be coordination-based, where intelligent decisions for resource allocation and interference mitigation are based on information exchange between macro and femto BSs. We briefly review such coordinationbased approaches available in the literature for interference management.

- Resource Partitioning based methods: The simplest of the resource partitioning mechanism is to allocate all resources to all femtocells. This is known as Frequency Reuse-1 or simply Reuse-1. Such resource allocation improves the resources utilization. However, it may increase the co-layer interference significantly when the density of femtocells increases. In [9-11], authors suggest Fractional Frequency Reuse (FFR) for OFDMA-based macro-femto network. They deploy hybrid spectrum allocation where orthogonal allocation is deployed for inner femtocells (located close to macro BS) and shared allocation for the outer femtocells (located away from macro BS). These schemes ensure cross-layer interference mitigation to the FUEs located near macro BS. Dynamic resource partitioning for cross-layer interference avoidance is proposed in [6], where femto BSs are denied access (via wired backhaul) to those resources that are assigned to nearby macro UEs. In [12], authors propose a low complexity randomized interference avoidance method for femtocells. Each femtocell is allocated a random subset of resources considering the fact that neighboring femtocells are unlikely to use the identical resources 
consistently. In [13], location based resource management algorithm is proposed which allows femtocell to reuse macrocell resources to increase spatial reuse. Authors provide a hashing scheme based resource allocation for femtocells which does not require coordination. In $[7,8,14,15]$, coordinationbased co-layer interference avoidance and adaptive frequency reuse (AFR) algorithms are proposed. It begins with orthogonal resource allocation to femtocells and then, coordination-based resource reuse is deployed to improve spectrum efficiency. The coordination between femtocells ensures that co-layer interference remains below the acceptable level. Due to the initial orthogonal allocation, such schemes suffer from poor resource utilization in the initial phase of algorithm.

- Transmit power control based methods: In [16] and [17], a power control method is suggested to achieve constant femto BS coverage while ensuring no adverse impact on the macrocell throughput. In [18], a reward (signal to interference ratio) and penalty (interference) based objective function is formulated for femto BSs in which the interfering femto $\mathrm{BSs}$ reduce their transmit power to mitigate crosslayer interference. Similar adaptive power control algorithms to mitigate cross-layer interference are discussed in [4] and [5]. In [19], two joint power control and resource allocation schemes are discussed, one is centralized while other is a coordination-based distributed scheme.

- Cognition based methods: Femtocells may determine interference pattern and resource utilization of network cognitively [20]. Authors consider femto BSs as secondary users, determine the available channels cognitively and design autonomous algorithms for cross-layer interference management. The benefits of cognitive approach depend on the spectrum occupancy of primary macrocell UEs.

- Self-organized and learning based methods: In [21], we have proposed a self-organized resource allocation algorithm to mitigate intercell interference in a macro-relay network. Focussing on macro-femto network in [22], resource allocation algorithm to avoid co-layer interference is executed at the backhaul after each femtocell identifies its neighboring femtocells. Authors in [23] propose sensing and tuning phase to minimize interference and maximize the system performance. One method is based on information exchange between femtocells and other, on measurement reports from users. Both schemes give improved performance compared to random allocation policy. Authors describe a self optimization framework to jointly optimize spectrum assignment and transmission power in [24] and Q-Learning based distributed interference control scheme for self-organized femtocell network in [25] to mitigate cross-layer interference.

In this paper, we propose a scheme, which mitigates colayer interference, while improving the minimum rate (10 percentile throughput) achieved by FUEs and ensuring fairness to them. This issue of improved minimum rate achieved by FUEs and fairness along with interference mitigation has not been addressed in the literature to the best of our knowledge.

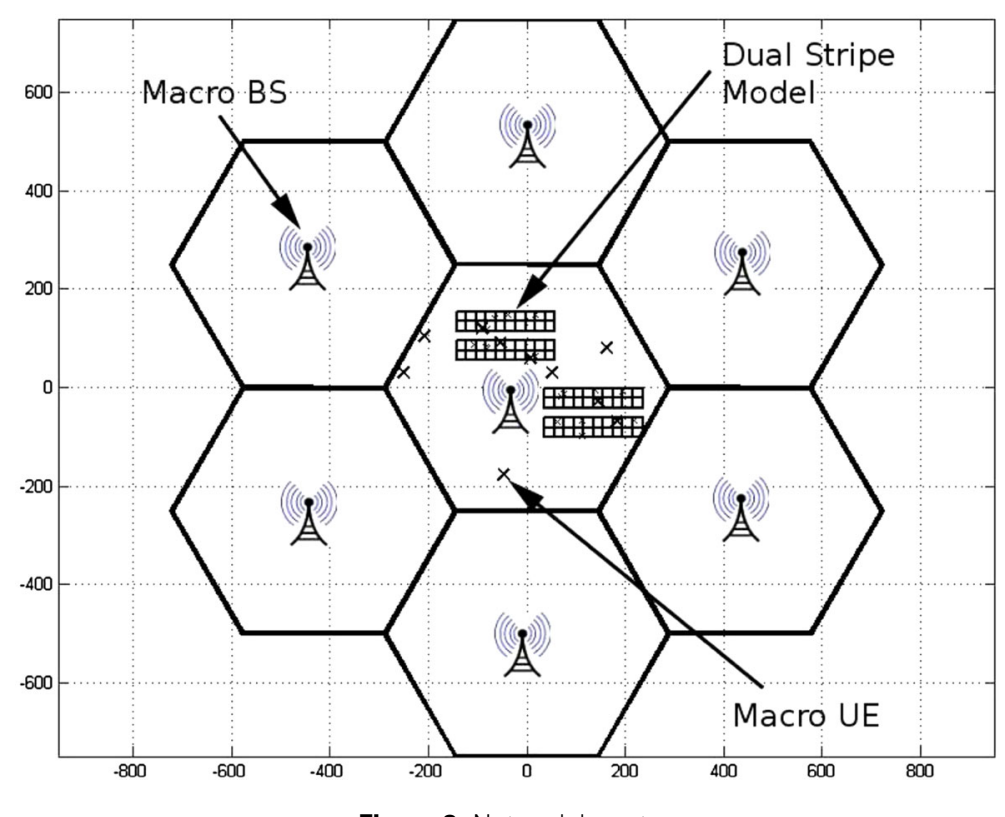

Figure 2. Network layout. 


\section{SYSTEM MODEL AND PROPOSED RESOURCE ALLOCATION SCHEMES}

\subsection{System model}

We consider the downlink transmission scenario in an OFDMA-based macro-femto network. Our system model consists of seven macrocells with macro BS located at the center of each macrocell. $L$ femtocells are overlaid in the central macrocell (Figure 2). In an OFDMA-based LTE network [26], the system resources are divided along frequency (subcarriers) and time slots. These resources are scheduled in units of physical resource blocks (PRBs). Each PRB (bandwidth $=180 \mathrm{kHz}$ ) consists of 12 subcarriers. We assume that $N$ PRBs are available for both macrocells and femtocells. To compute SINR, we use the path loss models for these links: between FUE and femto BS when FUE is in the same/different apartment as femto BS, and between FUE and macro BS when FUE is inside the apartment as specified in [27].

Next, we describe both of our proposed resource allocation schemes.

\subsection{Proposed AFR with power control scheme}

In this section, we illustrate our proposed modification to the existing AFR scheme, and we call it adaptive frequency reuse (AFR) with power control. The existing AFR scheme $[7,8,14,15]$ achieves coordination-based co-layer interference avoidance, where the neighboring femtocells use nonoverlapping resources initially. Later, femtocells attempt to reuse the resources by coordinating with neighbors to improve resource utilization efficiency. It is ensured that the co-layer interference does not exceed the tolerable threshold. However, its limitations are as follows: (i) initially, system performance is low due to inefficient resource utilization, and (ii) this scheme does not consider the reuse of resources at lower power.

Adaptive frequency reuse with power control is our proposed modification to the existing AFR scheme. When a femto BS cannot use a particular PRB at full transmit power, it is likely that the same PRB may be used with reduced power level without causing significant interference to its neighboring femtocells. We exploit this concept in AFR with power control scheme, which is a two-step interference coordination algorithm. In the first step, resources are shared between interfering femtocells in an orthogonal manner. Then in the second step, reuse of resources is facilitated with power control. When a femto BS is not allowed to use a PRB at full transmit power due to interference concerns, it checks for the feasibility of using the same PRB at half of the transmit power. This feasibility is determined by a-priori interference measurement, which may be caused to neighboring femtocells if this PRB was used. If this interference is less than the acceptable threshold, femto BS is allowed to use that PRB at half the transmit power. Note that the idea behind using half the transmit power is to exploit the possibility of maximizing throughput of femtocells by transmit power variation, which was not explored in the AFR scheme.

\subsection{Proposed self-organized resource allocation scheme}

There is an inevitable trade-off between aggressive resource reuse and co-layer interference. Our proposed self-organized resource allocation scheme meets this tradeoff by coordination between femtocells. It is a two-step algorithm to reduce co-layer interference between femtocells, while ensuring rate requirement satisfaction and fairness to FUEs.

In Step-1, interfering neighbor set for each femtocell is identified and Reuse-1 is employed. In Step-2, each femto BS identifies PRB, which offers minimum SINR and performs two levels of a-priori check to ensure that dropping of that PRB does neither cause any degradation in system performance nor in its own performance. The femto BS drops that identified PRB to reduce co-layer interference. This is performed iteratively for all femto BSs. This selforganized resource allocation algorithm mitigates co-layer interference by exchanging information with neighboring femtocells locally and achieves an overall improvement in system performance. Figure 3 gives the flowchart of SO-RA algorithm.

\section{Step-1: Interfering neighbor set discovery and initial Reuse-1 allocation}

In accordance with the LTE standard [28], reference signal received power measurement is performed by UEs for path loss estimation between UE and BS [26]. Based on these measurements made by FUE, femto BS computes path loss from neighboring femto BSs, which are then compared with specific threshold value $P L_{t h}$ to determine whether they may cause interference or not. Thus, each femto BS determines a set of neighboring femto BSs that are likely to cause significant interference to its FUEs. The interfering neighbor set of femto $\mathrm{BS} l$ is given by,

$$
\begin{aligned}
I_{l} & =\left\{\text { Femto } B S_{j} \mid P L_{j, l}-P L_{l, l}>P L_{t h}\right\}, \\
j & =1,2 \ldots L
\end{aligned}
$$

where $P L_{j, l}$ is pathloss between FUE $l$ and femto BS $j$ and $P L_{l, l}$ is pathloss between FUE $l$ and femto BS $l$. Note that same index $l$ is used for femto UE and femto BS because we consider only one femto UE per femtocell. Note that the underlying assumption in determining threshold value $P L_{t h}$ is that only those femtocells present in the vicinity cause co-layer interference.

After interfering neighbor set discovery, resource allocation is to be performed. Most of the existing schemes performs orthogonal allocation initially $([7,14,15])$. However, this increases the signaling overheads required for coordination in the initial stage, while in SO-RA, 


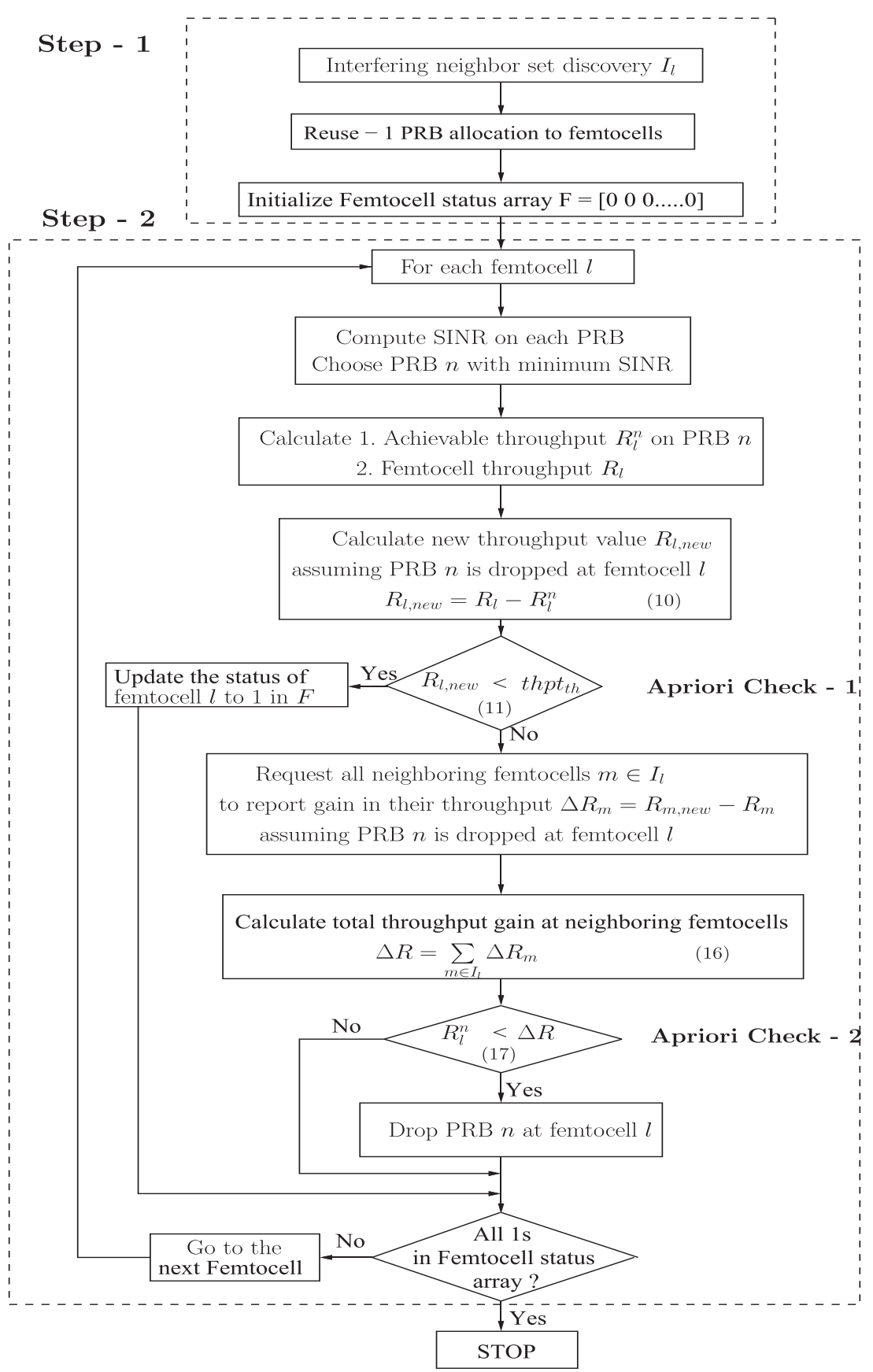

Figure 3. Self-organized resource allocation algorithm.

employing Reuse-1 eliminates the need of coordination between femto BSs at the initial stage and signaling overhead reduces.

\section{Step-2: Coordinated resource drop}

In this step, each femto BS identifies and drops the PRB with minimum SINR, such that neither femtocell throughput reduces below the threshold nor the overall system performance deteriorates. We assume that messages required for coordination are exchanged between femto BS and its interfering neighbors via backhaul. The SINR for FUE $l$ on PRB $n$ is given by,

$$
S I N R_{l}^{n}=\frac{P_{F t x}^{l, n} \cdot P L_{l, l}}{I_{\text {femto }}+I_{\text {macro }}+N_{o}}
$$


where

$$
I_{\text {femto }}=\sum_{j=1, j \neq l}^{\left\|I_{l}\right\|} P_{F t x}^{j, n} \cdot P L_{j, l} \cdot x_{j, n}
$$

and

$$
I_{\text {macro }}=\sum_{k=1}^{7} P_{M t x}^{k, n} \cdot P L_{k, l}^{\prime} \cdot y_{k, n} .
$$

$P_{F t x}^{j, n}$ and $P_{M t x}^{k, n}$ denote the transmit power of femto BS $j$ and macro BS $k$ on PRB $n$, respectively. $P L^{\prime}{ }_{k, l}$ is the pathloss between FUE $l$ and macro BS $k . x_{j, n}$ is a variable indicator that denotes whether PRB $n$ is used by femto BS $j$ or not. Similarly, $y_{k, n}$ indicates whether PRB $n$ is used by macro BS $k$ or not. $N_{o}$ is additive white Gaussian noise.

To determine which PRB to drop, femto BS $l$ calculates the SINR experienced on all PRBs that are used by its FUEs. Then, it chooses a candidate PRB $n$ on which it experiences minimum SINR. It is likely that minimum SINR is due to high amount of interference on that PRB. If such PRB is dropped, it may reduce the interference caused to the neighboring femtocells, at the cost of reduced serving femtocell throughput. To ensure that this penalty is minimal, we select the PRB with minimum SINR, so that its dropping results in minimum rate loss and eventually co-layer interference reduces. However, to ensure that the decision of dropping PRB (taken in coordination with the localized neighborhood) does not adversely affect the global system performance, femto BS $l$ performs two levels of a-priori checks before actually dropping that PRB.

(1) Level-I a-priori check:

Here, we analyze the impact of dropping PRB $n$ on the performance of serving femtocell $l$. For femto $\mathrm{BS} l$, the achievable throughput on $\operatorname{PRB} n$ is given by,

$$
R_{l}^{n}=B \cdot \log _{2}\left(1+\operatorname{SINR} R_{l}^{n}\right)
$$

and total throughput of femto BS $l$ is given by,

$$
R_{l}=\sum_{n=1}^{N} B \cdot \log _{2}\left(1+\operatorname{SINR}_{l}^{n}\right) \cdot x_{n, l}
$$

where $B$ is the PRB bandwidth.

Femto BS $l$ calculates its new throughput value $R_{l, \text { new }}$ assuming that it has dropped $\operatorname{PRB} n$ as,

$$
R_{l, \text { new }}=R_{l}-R_{l}^{n}
$$

Further, it compares the new throughput $R_{l, \text { new }}$ with the specified threshold thpt $t_{t h}$. If

$$
R_{l, \text { new }}<\text { thpt }_{\text {th }}
$$

then femto BS defers the decision to drop PRB $n$ and algorithm repeats for the next femtocell. Otherwise, when (8) is not satisfied, it implies that Level-I a-priori Check results in favor of dropping PRB $n$. Only then, femto BS $l$ initiates Level-II a-priori Check, as discussed next.

(2) Level-II a-priori check:

Here, we analyze the impact of dropping PRB $n$ on the performance of neighboring femtocells by coordination. Femto BS $l$ requests all its neighboring femto BSs to report the gain in their individual throughputs assuming femto BS $l \in I_{l}$ has dropped PRB $n$. Each femto BS $m \in I_{l}$ calculates its new SINR and throughput as follows,

$$
S I N R_{m, \text { new }}^{n}=\frac{P_{F t x}^{m, n} \cdot P L_{l, l}}{I_{\text {femto }}-P_{F t x}^{l, n} \cdot P L_{m, l}+I_{\text {macro }}+N_{o}}
$$

where

$$
\begin{gathered}
I_{\text {femto }}=\sum_{j=1, j \neq m}^{\left\|I_{m}\right\|} P_{F t x}^{j, n} \cdot P L_{j, m} \cdot x_{j, n} . \\
R_{m, \text { new }}=\sum_{n=1}^{N} B \cdot \log _{2}\left(1+\operatorname{SINR}_{m, \text { new }}^{n}\right) \cdot x_{n, l}
\end{gathered}
$$

Then, femto BS $m$ computes gain in throughput as,

$$
\Delta R_{m}=\sum_{m \in I_{l}}\left(R_{m, \text { new }}-R_{m}\right)
$$

On receiving $\Delta R_{m}$ from all neighboring femto BSs, femto BS $l$ calculates the total throughput gain of its neighbors as,

$$
\Delta R=\sum_{m \in I_{l}} \Delta R_{m}
$$

To observe the impact of dropping PRB $n$ on the overall system throughput, we compare,

$$
R_{l}^{n}<\Delta R
$$

where $R_{l}^{n}$ represents the loss in throughput due to dropping PRB $n$ and $\Delta R$ represents the net gain in system throughput.

If dropping PRB $n$ at femto BS $l$ results in increasing system throughput (3.4), femto BS $l$ takes a final decision to drop the PRB $n$. Otherwise, Step-2 is repeated for the next femto $\mathrm{BS}$.

Level-I check provides minimum throughput guarantee to each Femto cell, while Level-II check allows the femtocell throughput to increase further (above the minimum threshold) to the extent that the neighboring femtocells are not adversely affected.

Step- 1 of the algorithm is triggered periodically after a predetermined number of OFDMA frames. This repetition period can be configured based on the system dynamics. 
Step- 2 of the algorithm gets implemented iteratively for each femto BS successively. The algorithm stops when any further dropping of PRB becomes infeasible due to violation of minimum throughput guarantee to the femto BS.

In case any femto BS gets deactivated during Step-2, it will release its PRBs, and they will become available for reuse at its neighboring femto BSs immediately. However, Step-2 of our algorithm does not consider reallocation of resource and therefore, until Step-1 is revoked, resources may remain underutilized. However, due to the periodic repetition of Step-1, underutilization of resources is likely to happen only for a small time duration.

The distinct features of SO-RA scheme are the following:

- It ensures minimum throughput guarantee to the FUEs by applying Level-I a-priori check.

- In Level-II a-priori Check, each femto BS decides to drop PRB by comparing the impact of dropping that PRB on self and neighbors. This distributed decision making based on localized interaction between neighboring femto BSs makes algorithm self-organized.

- Our algorithm iteratively drops the PRB with minimum SINR such that the throughput requirement of FUEs is ensured and overall gain is not compromised. It also helps in reducing interference to MUEs located either close to femto BS or in the overlapping coverage area. Thus, SO-RA scheme reduces cross-layer interference implicitly.

\subsection{Comparative summary}

In this section, we highlight the key aspects of both of our proposed schemes, proposed AFR with power control and SO-RA, in comparison with the state-of-the-art, Reuse-1 and AFR scheme. Note that we focus only on the resource allocation for femtocell users.

In Reuse-1, all resources are available to all femtocells, and there is no restriction on their usage. As mentioned before, this improves the resource utilization, while compromising on the co-layer interference. In AFR scheme, resource allocation begins with assignment of disjoint resources to each femtocell and then, reuse of resources is permitted with appropriate coordination amongst the neighboring femtocells. The decision for reuse of resource is based on whether the co-layer interference lies below the acceptable threshold limits or not.

In our proposed modification to the AFR scheme, we go a step further and give consideration to the reuse of resource at lower power level, if the reuse is not feasible at the maximum transmit power. We perform a-priori interference measurement to ensure that the co-layer interference remains below the acceptable threshold limits when the resources are reused at lower power level.

In case of our proposed SO-RA scheme, we intend to maximize the resource utilization, like Reuse- 1 scheme and reduce co-layer interference simultaneously. The allocation strategy is to begin with reuse- 1 amongst the most interfering set of femtocell neighbors. Then, the next step is to identify that resource on which femtocell user is experiencing the worst SINR, i.e., identify the most interfering PRB. Now, we drop this PRB after ensuring that dropping does not impact the femtocell performance and the overall network performance. This is performed iteratively for all femtocells in the network.

Thus, in case of our proposed modification to AFR, we attempt to gradually maximize the resource reuse and exploit the power dimension to reduce co-layer interference. In contrast, with SO-RA, we begin with aggressive Reuse- 1 and then, selectively drop the most interfering $\mathrm{PRBs}$, in a iterative manner to reduce co-layer interference. The interesting aspect of SO-RA is that with the change in user statistics like new users joining the network or existing ones exit. However, the algorithm of our proposed SO-RA scheme introduces computational complexity. Also, this algorithm takes some finite time to converge. The convergence time analysis can be investigated as future work.

\section{SIMULATION RESULTS}

To evaluate the performance of our proposed schemes, we have performed system level simulations in MATLAB. The simulation parameters are given in Table I. We have carried out performance analysis for the users located in the central cell. For the FUEs, we consider the interference from all seven macro cells and the neighboring femtocells.

We consider dual stripe model for dense urban femtocell deployment where each femtocell block has two stripes of apartments [29]. Each stripe has $2 \times 10$ apartments each of size $10 \mathrm{~m} \times 10 \mathrm{~m}$. To ensure sufficient separation between femto BSs from different stripes, there is a $10 \mathrm{~m}$ wide street in between and a $10 \mathrm{~m}$ wide space around the stripe (Figure 4). Each femtocell can have random number of floors $F$ within the range 1 to 10 . So each femtocell block has a $40 \times \mathrm{F}$ apartments. Each apartment may not have

Table I. Simulation parameters.

\begin{tabular}{lc}
\hline Parameter & Value \\
\hline Inter site distance & $500 \mathrm{~m}$ \\
Total bandwidth & $10 \mathrm{MHz}$ with 50 PRBs \\
PRB bandwidth & $180 \mathrm{KHz}$ \\
Macro users per macrocell & 10 \\
Number of femtocell blocks & 3 \\
Deployment ratio $\beta$ & 0.2 \\
Active ratio $\rho$ & $0.1 \mathrm{to} 1$ \\
Macro BS TX power & $46 \mathrm{dBm}$ \\
Femto BS TX power & $20 \mathrm{dBm}$ \\
Macro BS antenna gain & $14 \mathrm{dBi}$ \\
Femto BS antenna gain & $0 \mathrm{dBi}$ \\
UE noise figure & $9 \mathrm{~dB}$ \\
Wall losses $L_{\text {ow }}, L_{\text {ow }, 1}$ & $20 \mathrm{~dB}, 5 \mathrm{~dB}$ \\
Pathloss threshold $P L_{t h}$ & $30 \mathrm{~dB}$ \\
Femto BS throughput & $7 \mathrm{Mbps}$ \\
threshold thpt & \\
Averaging interval & $1000 \mathrm{~ms}$ \\
\hline
\end{tabular}




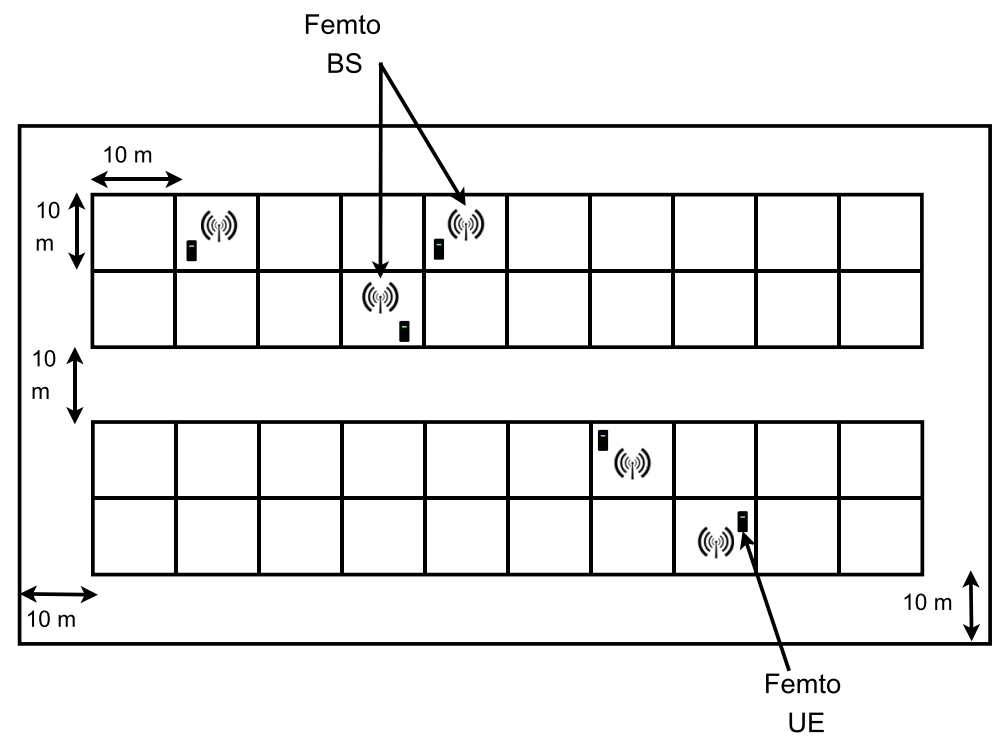

Figure 4. Dual Stripe Model for Femtocell Deployment.

active femto BS in it. To model this scenario, two ratios are defined: deployment ratio $(\beta)$ indicates the fraction of apartments with femto BS, and active ratio $(\rho)$ gives the fraction of active femto BSs.

We assume that each apartment can have at most one active femto BS in it, located at the center of apartment. Both FUEs and MUEs are dropped uniformly in the apartment and macrocell, respectively. We assume only one floor per femtocell block, that is, $F=1$. We consider only one FUE per femtocell and therefore, use same indices for FUE and femto BS. Also, we assume femto BSs to operate in closed access mode (i.e., only a set of users are allowed association with femto BS). We consider four femtocell blocks with deployment ratio $\beta=0.2$ and active ratio $\rho$ varying from 0.1 to 1 . This implies that there are $4 \times(20 \times 0.2)=16$ femtocells deployed in the network, of which a set of femtocells will be active based on the active ratio. To model the scenario of randomly deployed femtocells, we have performed the following. For a given active ratio, we have randomly varied the femto block position, femtocell user location in that block and macro UE locations. Then, all simulations are averaged over an interval of $1000 \mathrm{~ms}$

We review the significance of femtocell deployment by comparing the throughput performance of users with and without femtocell deployment in Figure 5. We observe that about $80 \%$ of the users experience an improvement of more than $5 \mathrm{Mbps}$ in their throughput and $30 \%$ of the users experience an improvement of more than $10 \mathrm{Mbps}$, when femtocells are deployed in the network. To investigate the impact of increased femtocell density (i.e., active ratio) on the cell throughput performance of macro and femto cells, we consider four femtocell blocks with deployment ratio $\beta=1$ and active ratio $\rho$ varying from 0.1 to 1 . We observe from Figure 6 that with an increase in the femto cell density, the femtocell users experiences increased co-layer

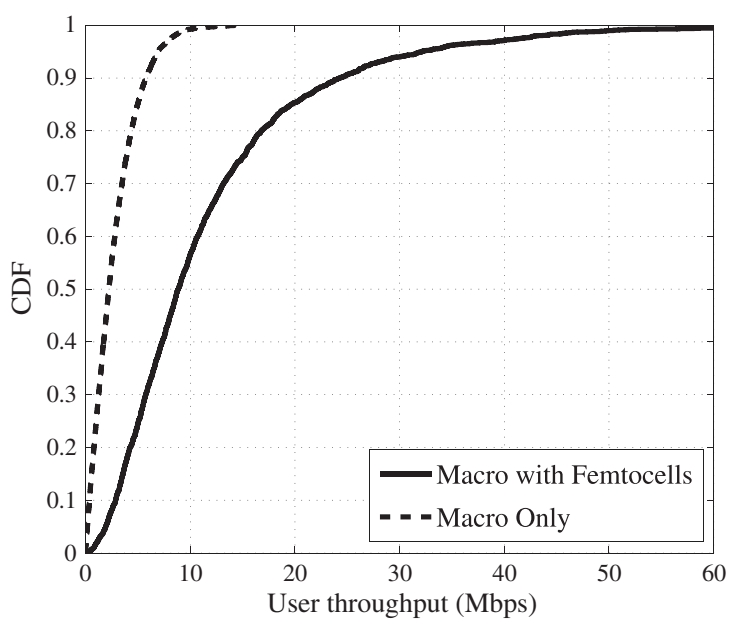

Figure 5. Performance comparison-with and without femtocell.

interference while macrocell users experience increased cross-layer interference. As a consequence, the average throughput in both reduces by about $57 \%$.

Figure 7 compares the performance of SINR per PRB for both of our proposed schemes, AFR with power control, and SO-RA scheme with that of Reuse-1 and AFR scheme. We observe that the SINR performance of AFR scheme is the best of all. This happens because of the fact that resource allocation in AFR begins with orthogonal allocation, which is followed by co-channel reuse subject to condition that the co-layer interference remains less than the acceptable threshold. because of this constrained resource allocation, majority of the allocated PRBs are likely to be orthogonal with respect to those PRBs that are allocated to other femtocell users. However, this reduces 


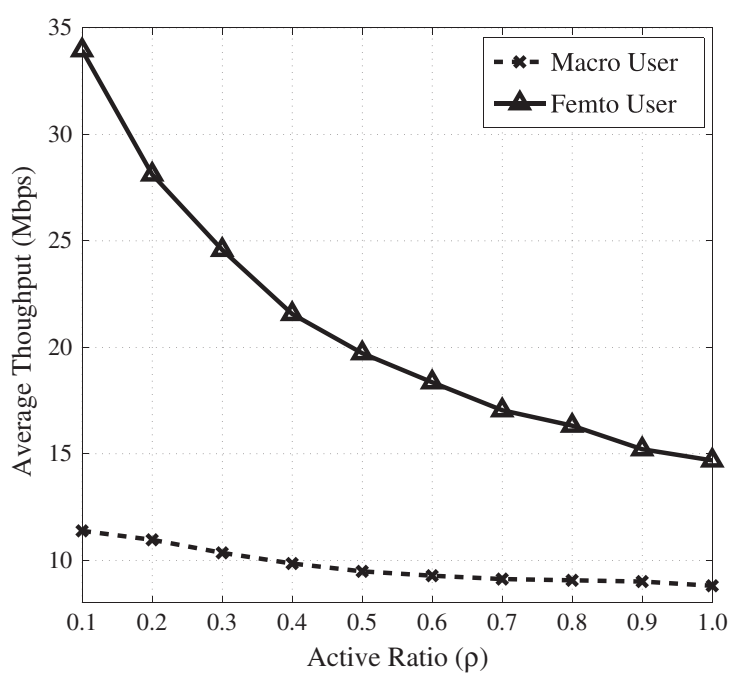

Figure 6. Impact of active ratio on throughput.

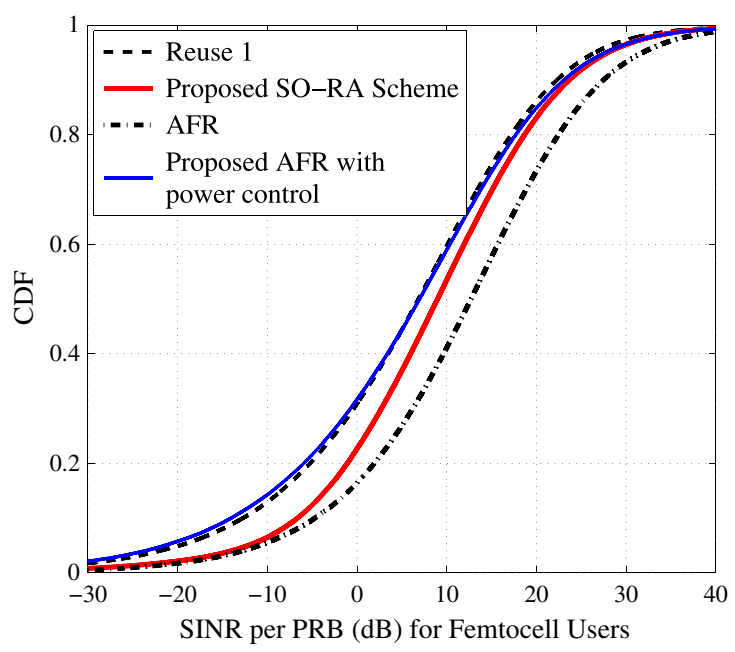

Figure 7. SINR per PRB for Femtocell users.

the resource utilization by about $60 \%$ in case of AFR scheme as shown in Table II. We also observe that the performance of Reuse-1 and proposed AFR with power control is almost similar. This happens because we increase the reuse of resources in proposed AFR by relaxing the full power allocation constraint of the AFR scheme. As a result, we observe $89 \%$ resource utilization in proposed AFR with power control compared to $41 \%$ achieved in case of AFR scheme. Note that the cummulative ditribution function (CDF) of SINR per PRB for the proposed SO-RA scheme falls in between Reuse-1 and AFR. Also, the resource utilization in proposed SO-RA scheme is $83.7 \%$, which is almost two times of that obtained using AFR scheme. This indicates that our proposed SO-RA scheme achieves tradeoff in maximizing user throughput and improving resource utilization.

We compare the performance of our proposed SO-RA scheme with Reuse-1, AFR, and AFR with power control
Table II. Resource utilization efficiency of different schemes.

\begin{tabular}{lc}
\hline $\begin{array}{l}\text { Frequency allocation } \\
\text { scheme }\end{array}$ & $\begin{array}{c}\text { Resource utilization } \\
\text { efficiency }\end{array}$ \\
\hline Reuse-1 & $100 \%$ \\
AFR & $41.3 \%$ \\
Proposed AFR & $89 \%$ \\
with power control & $(41.3 \%$ resources with full \\
& power and $47.7 \%$ resources \\
Proposed SO-RA scheme & at half power) \\
\hline
\end{tabular}

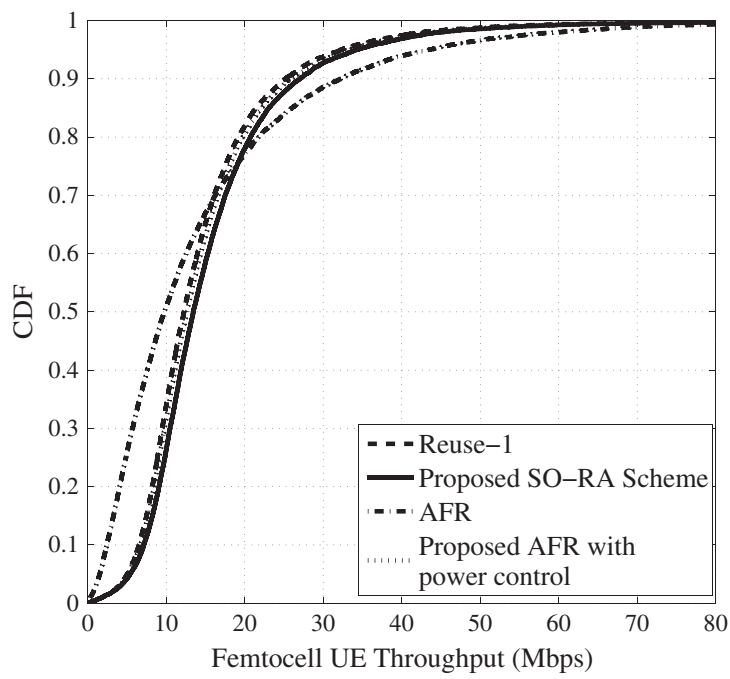

Figure 8. CDF of femtocell UE throughput.

Table III. Throughput comparison of different schemes.

\begin{tabular}{lcc}
\hline & \multicolumn{2}{c}{ FUE throughput (Mbps) } \\
\cline { 2 - 3 } $\begin{array}{l}\text { Frequency allocation } \\
\text { scheme }\end{array}$ & $\begin{array}{c}\text { Avg. } \\
\text { throughput }\end{array}$ & $\begin{array}{c}\text { 10 percentile } \\
\text { throughput }\end{array}$ \\
\hline Reuse-1 & 28.83 & 13.05 \\
AFR & 29.57 & 10.6 \\
Proposed AFR & & \\
with power control & 29.21 & 13.18 \\
Proposed SO-RA scheme & 29.92 & 13.79 \\
\hline
\end{tabular}

scheme [7]. Figure 8 compares the cumulative distribution functions of average throughput of FUEs. For better understanding, Table III gives the average and 10 percentile throughput comparison of all four schemes. There is a slight improvement in the average throughput performance of SO-RA scheme compared to all other schemes. It is observed that SO-RA achieves 30\% improvement in the 10 percentile throughput performance of FUEs compared to AFR scheme. This happens due to the following reason - in SO-RA scheme, the femto BS experiencing severe interference does not drop PRBs simply if it deteriorates its own performance (ensured by Level-I a-priori 


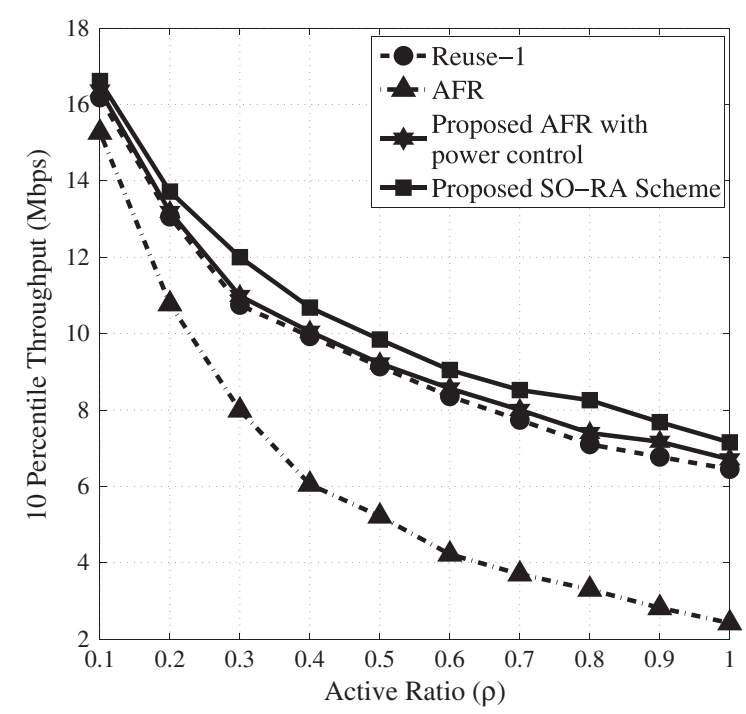

Figure 9. 10 percentile Throughput comparison of different schemes.

check). Rather, SO-RA strategy ensures that the loss in throughput performance of femto BS is lesser than the net gain in throughput performance of neighboring femto BSs. Thus, global system performance improvement in SO-RA scheme is ensured. On the contrary, to increase the system throughput performance, the severely interfered femto BS in AFR scheme may allow neighboring femto BSs to reuse PRBs, thereby increasing co-layer interference. In AFR with power control scheme, reuse efficiency improves at the cost of reduced throughput. However, the average throughput performance of AFR with power control is close to that of AFR without power control, but the 10 percentile throughput performance shows an improvement of about $24 \%$ relatively.

Figure 9 shows the impact of increased femtocell density on the 10 percentile throughput of FUEs for different schemes. SO-RA scheme offers the best 10 percentile throughput, even with increased femtocell density. The performance of AFR with power control is close to Reuse-1 with marginal improvement when the femtocell density increases.

Further, we investigate the fairness performance (Figure 10) by using Gini fairness index. It is given by,

$$
I=\frac{1}{2 L^{2} \bar{R}} \sum_{l=1}^{L} \sum_{m=1}^{L}\left|R_{l}-R_{m}\right|
$$

where $\bar{R}=\frac{\sum_{l=1}^{L} R_{l}}{L}$. Gini fairness index lies between 0 and 1. A scheme is perfectly fair if Gini index is 0 and unfair if 1 . We observe that SO-RA scheme outperforms in terms of fairness to FUEs. AFR with power control exhibits similar performance behavior as for the 10 percentile throughput (Figure 9).

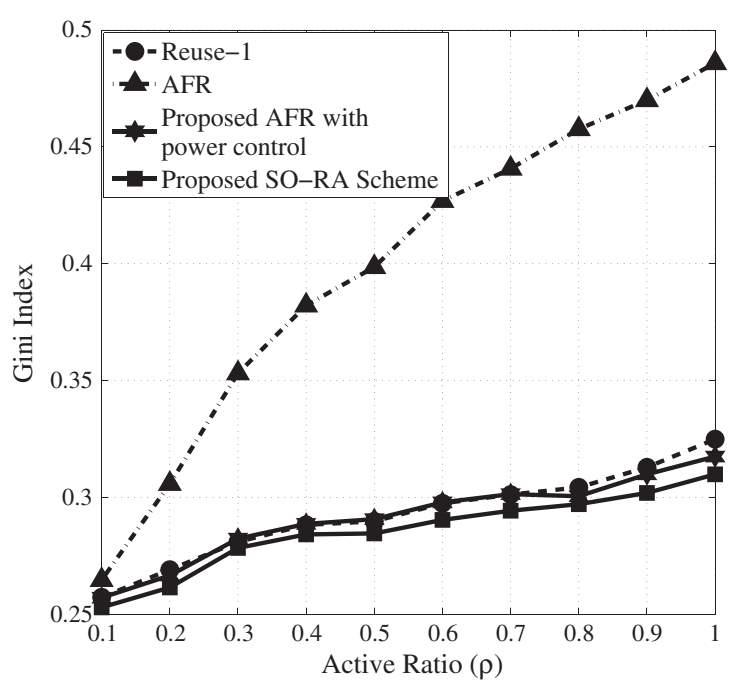

Figure 10. Comparison of Gini fairness index.

Finally, we compare the schemes based on the resource utilization efficiency metric (Table II). We define resource utilization efficiency $\left(\eta_{r u}\right)$ as the ratio of used PRBs to the available PRBs and is given by,

$$
\eta_{r u}=\frac{\sum_{j=1}^{F_{\text {num }}} P R B_{j}}{P R B_{\text {tot }} \times F_{\text {num }}}
$$

where $F_{\text {num }}$ gives the count of active femtocells in the network. $P R B_{j}$ and $P R B_{\text {tot }}$ denotes the number of used PRBs in $j^{\text {th }}$ femtocell and total number of PRBs available in the system, respectively.

The resource utilization efficiency is the lowest for AFR. $\eta_{r u}$ in AFR with power control scheme is close to Reuse1. However, an important observation is that only $41.3 \%$ of resources are used with full transmit power and remaining $47.7 \%$ resources are used at half of the transmit power. In SO-RA scheme, $\eta_{r u}$ gets almost doubled compared to AFR, with all resources being used at full transmit power.

In a nutshell, our results indicate improved 10 percentile throughput and fairness performance in SO-RA scheme compared to AFR with power control, AFR, and Reuse1 schemes. Thus, SO-RA scheme offers a reasonable trade-off in achieving improved throughput performance of severely affected FUEs, fairness to all FUEs, and improved resource utilization efficiency.

\section{CONCLUSIONS}

Femtocell deployment provides capacity and coverage improvement to indoor users. However, intelligent and self-organized resource allocation is required to ensure improved performance with minimal interference and QoS guarantees. In this paper, we have proposed two resource allocation algorithms. First, proposed AFR with power control scheme performs at par with that of Reuse-1 in terms of average throughput and resource utilization 
efficiency. Second, proposed self-organized resource allocation algorithm reduces co-layer interference in the downlink scenario, while ensuring throughput performance improvement to the severely affected FUEs and improvement in resource utilization and fairness to all FUEs simultaneously. Thus, SO-RA scheme achieves feasible trade-off between 10 percentile throughput, fairness and resource utilization efficiency, compared to other schemes available in the literature. The two levels of a-priori check in SORA operate in a self-organized manner to ensure that the emphasis is not on the localized performance improvement of an individual femtocell, but on the global system performance improvement.

\section{ACKNOWLEDGEMENT}

This project is being carried out under the India-UK Advanced Technology of Centre (IU-ATC) of Excellence in Next Generation Networks project and funded by the Department of Science and Technology (DST), Government of India, and UK EPSRC Digital Economy Programme.

\section{REFERENCES}

1. Chandrasekhar V, Andrews JG, Gatherer A. Femtocell Networks: A Survey. IEEE Communications Magazine 2008; 46(9): 59-67.

2. Zhang J, de la Roche G. Femtocells: Technologies and Deployment. John Wiley \& Sons: United Kingdom, 2010.

3. Perez L, Valcarce D, Roche AD, Zhang GJ. OFDMA Femtocells: A Roadmap on Interference Avoidance. IEEE Communications Magazine 2009; 47(9): 41-48.

4. Kim MS, Je HW, Tobagi F. Cross-tier Interference Mitigation for Two-tier OFDMA Femtocell Networks with Limited Macrocell Information. In IEEE Global Telecommunications Conference (GLOBECOM), Miami, FL, 2010; 1-5.

5. Morita M, Matsunaga Y, Hamabe K. Adaptive Power Level Setting of Femtocell Base Stations for Mitigating Interference with Macrocells. In IEEE Vehicular Technology Conference, Ottawa, ON, 2010; 1-5.

6. Bharucha Z, Saul A, Auer G, Haas H. Dynamic resource partitioning for downlink femto-to-macrocell interference avoidance. In EURASIP Journal on Wireless Communications and Networking, 2010; 77-88.

7. Ruihong A, Xin Z, Gen C, Ruiming Z, Lin S. Interference avoidance and adaptive fraction frequency reuse in a hierarchical cell structure. In IEEE Wireless Communications and Networking Conference (WCNC), Sydney, Australia, 2010; 1-5.

8. Hu F, Zheng K, Lei L, Wang W. A distributed inter-cell interference coordination scheme between femtocells in LTE-advanced networks. In IEEE Vehicular Technology Conference, Yokohama, 2011; 1-5.

9. Bai Y, Zhou J, Liu L, Chen L, Otsuka H. Resource Coordination and interference mitigation between macrocell and femtocell. In IEEE Personal Indoor and Mobile Radio Communications Conference, Tokyo Japan, 2009; 1401-1405.

10. Guvenc I, Jeong MR, Watanabe F, Inamura H. A hybrid frequency assignment for femtocells and coverage area analysis for co-channel operation. In IEEE Communication Letters, vol. 12, 2008; 880-882.

11. Juang RT, Ting P, Lin HP, Lin DB. Interference management of femtocell in macro-cellular networks. In Wireless Telecommunications Symposium, Tampa, US, 2010; 1-4.

12. Chandrasekhar V, Andrews J. Spectrum allocation in tiered cellular networks. IEEE Transactions on Communications 2009; 57(10): 3059-3068.

13. Sundaresan K, Rangarajan S. Efficient resource management in OFDMA femtocells. In Mobile Ad hoc Networking and Computing (MobiHoc), New Orleans, LA, USA, 2009; 33-42.

14. Cao G, Yang D, Ruihong A, Xuan Y, Zheng R, Zhang $X$. An adaptive sub-band allocation scheme for dense femtocell environment. In IEEE Wireless Communications and Networking Conference, Cancun, Quintana Roo, 2011; 102-107.

15. Zheng K, Hu F, Lei Lei, Wang W. Interference coordination between femtocells in LTE-advanced networks with carrier aggregation. In International ICST Conference on Communications and Networking in China, Beijing, China, 2010; 1-5.

16. Jo HS, Mun C, Moon J, Yook JG. Self-optimized coverage coordination in femtocell networks. IEEE Transactions on Wireless Communications 2010; 9(10): 2977-2982.

17. Claussen H. Performance of macro- and co-channel femtocells in a hierarchical cell structure. In Personal, Indoor, Mobile Radio Communications, Athens, Greece, 2007; 1-5.

18. Chandrasekhar V, Andrews J, Muharemovic T, Shen $\mathrm{Z}$, Gatherer A. Power control in two-tier femtocell networks. IEEE Transactions on Wireless Communications 2009; 8(8): 4316-4328.

19. Cao G, Yang D, Ye X, Zhang X. A downlink joint power control and resource allocation scheme for cochannel macrocell-femtocell networks. In IEEE Wireless Communications and Networking Conference, Cancum, Quintana Roo, 2011; 281-286.

20. Cheng SM, Ao WC, Tseng FM, Chen KC. Design and analysis of downlink spectrum sharing in two-tier cognitive femto networks. IEEE Transactions on Vehicular Technology 2012; 61(5): 2194-2207. 
21. Mehta M, Aliu OG, Karandikar A, Imran MA. A selforganized resource allocation using inter-cell interference coordination (icic) in relay-assisted cellular networks. ICTACT Journal on Communication Technology 2011; 2(2): 300-313.

22. Liang YS, Chung WH, Ni GK, Chen IY, Zhang H, Kuo $S Y$. Resource allocation with interference avoidance in OFDMA femtocell Networks. IEEE Transactions on Vehicular Technology 2012; 61(5): 2243-2255.

23. Lopez-Perez D, Ladanyi A, Juttner A, Zhang J. OFDMA femtocells: A self-organizing approach for frequency assignment. In IEEE Personal, Indoor and Mobile Radio Communications, Tokyo, Japan, 2009; 2202-2207.

24. Bernardo F, Agusti R, Cordero J, Crespo C. Selfoptimization of spectrum assignment and transmission power in OFDMA femtocells. In Advanced International Conference on Telecommunications, Barcelona, 2010; 404-409.

25. Galindo-Serrano A, Giupponi L. Distributed Qlearning for interference control in OFDMA-based femtocell networks. In IEEE Vehicular Technology Conference, Taipei, 2010; 1-5.

26. Agilent. 3GPP long term evolution: system overview, product development, and test challenges, June 2009.

27. 3GPP TR 36814. Further advancements for E-UTRA physical layer aspects V900, March 2010.

28. 3GPP TR 36921. FDD home eNodeB (HeNB) radio frequency $(\mathrm{RF})$ requirements analysis V1000, April 2011.

29. Femtoforum. Interference management in OFDMA femtocells. http://smallcellforum.org [accessed on March 2010].

\section{AUTHORS' BIOGRAPHIES}

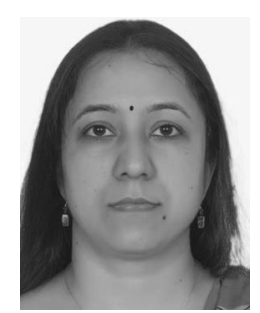

Mahima Mehta received the B.E. degree in Electronics and Communication Engineering from Government Engineering College, Bhopal, India, in 2001; the M.E. degree in Electronics and Communication Engineering from the Shri Govindram Seksaria Institute of Technology and Science, Indore, India, in 2007; and the Ph.D. degree in Electrical Engineering from the Indian Institute of Technology (IIT) Bombay, Mumbai, India, in 2014. She has worked as a Lecturer and then as an Assistant Professor for about eight years and has research experience of about five years. She is currently a project Research Scientist with IIT Bombay. She has been actively involved in a DST-funded project: India-UK Advanced Technology Centre (IU-ATC) and collaborates with researchers in UK and India. She is also a 3GPP RAN2 delegate. Her research interests include radio resource and mobility management in LTE heterogeneous cellular networks and LTE-WLAN interworking.

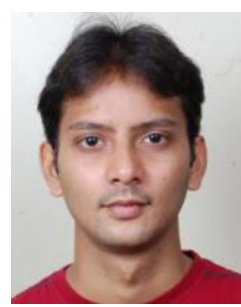

Nirbhay Rane received his B.E. Degree in Electronics and Telecommunication from Mumbai University in 2006. He completed his M.Tech. in Communication Engineering from the Indian Institute of Technology (IIT) Bombay in 2012. He worked as Research Associate student in 'Tata Indicom Center of excellence in Telecommunication Lab', IIT Bombay' from 2009-2012. His research interest lies mainly in Wireless networking, Mobile communication systems. He is currently working as Network Consulting Engineer in market leading networking company.

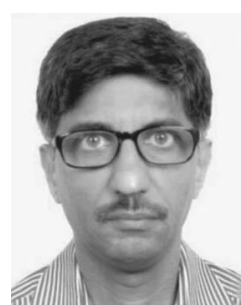

Abhay Karandikar earned his MTech and $\mathrm{PhD}$ degrees in Electrical Engineering from the Indian Institute of Technology, Kanpur in 1988 and 1994, respectively. He joined the Department of Electrical Engineering, IIT Bombay in April 1997, where he is currently Professor and Head of the Department. He is also the coordinator of Tata Teleservices IIT Bombay Center for Excellence in Telecom where he has initiated many projects in the area of broadband wireless networks. Dr Karandikar has several patents issued/pending, contributions to IEEE standards, contributed chapters in books and large number of papers in international journals and conferences to his credit. He has also served as technology advisor and consultant to many companies. He has served on the working group of telecom for 12th plan of Government of India. Dr Karandikar is a two time recipient of award for excellence in teaching at IIT Bombay- in 2006 and 2011.

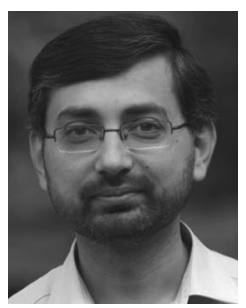

Muhammad Ali Imran received the M.Sc. (with Distinction) and Ph.D. degrees from Imperial College London, London, U.K., in 2002 and 2007, respectively. He is currently a Reader with the Centre for Communication Systems Research (CCSR), University of Surrey, Guildford, U.K., where he is leading a number of multimillion international research projects encompassing the areas of energy efficiency, fundamental performance limits, sensor networks, and selforganizing cellular networks. He is also leading the new physical-layer work area for $5 \mathrm{G}$ innovation center at Surrey (the 5G innovation center and an outdoor cellular test bed is being developed at Surrey University with a research grant of above 35 million). He has a global collaborative research network spanning both academia and key industrial players in the field of wireless communications. He has supervised 17 successful $\mathrm{Ph} . \mathrm{D}$. graduates and published over 150 peerreviewed research papers, including more than 25 IEEE Journals. His research interests include the derivation of 
information-theoretic performance limits, energy-efficient design of cellular systems, and learning/self-organizing techniques for the optimization of cellular system operation. Dr. Imran is a Fellow of the Higher Education Academy, U.K. He received the 2014 IEEE Communications Society Fred W. Ellersick Prize.

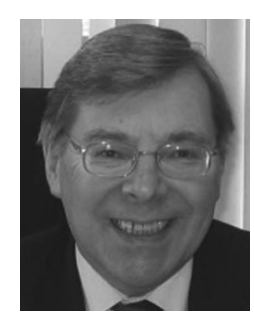

Barry G. Evans received the B.Sc. and Ph.D. degrees. Up until 2009, he was the Pro Vice Chancellor for Research and Enterprise with the University of Surrey, Guildford, U.K., and the Director of the Centre for Communication Systems Research, which is a 150 -strong postgraduate center. He now runs a number of research projects in satellite communications with the European Space Agency (ESA) and industry and leads the Surrey part of the UK-India network of excellence in next-generation networks. He is also the Director of a Surrey spin-off company MulSys Ltd. He has personal research background in satellite and mobile communications, working on speech coding, radio propagation, advanced physical layers, and cognitive radio with over 700 research publications and three textbooks to his name. Dr. Evans is the Editor of the International Journal of Satellite Communications and the Chair of the Steering Committee of SatNEx, which is an EU/ESA network of excellence, and the R\&D strategy group of the NETWORLD2020 satellite working group. He is a Fellow of the Royal Academy of Engineering, the Institution of Engineering and Technology, and the Royal Society of Arts and is a Chartered Engineer. 